\title{
The Domino Effect-Treatment of Superior Vena Cava Obstruction Triggering Tumor Lysis Syndrome: A Case Report
}

\author{
Suman Ghosh ${ }^{1}$, Tilak TVSVGK ${ }^{2 *}$, Venkatesan Somasundaram ${ }^{3}$, Mutreja Deepti ${ }^{4}$ \\ ${ }^{1}$ Resident (Medicine), Department of Internal Medicine, Armed Forces Medical College (AFMC), Pune, India. \\ ${ }^{2}$ Prof (Medicine) \& Med Oncologist, Department of Internal Medicine, Armed Forces Medical College (AFMC), Pune, India. \\ ${ }^{3}$ Prof (Pathology) \& Oncopathologist, Army Hospital (R\&R), Delhi Cantt, India. \\ ${ }^{4}$ Prof (Pathology) \& Molecular Pathologist, Department of Pathology, Armed Forces Medical College (AFMC), Pune, India.
}

Received 22 November 2020; Revised 19 February 2021; Accepted 26 February 2021; Published 01 March 2021

\begin{abstract}
Oncological emergencies present in a multitude of manners-structural, metabolic, hematologic, etc. affecting multiple systems, often. Urgent institution of therapy is often required for a successful outcome. Occasionally, the treatment of one emergency can initiate a related or unrelated emergency, necessitating management of all the complications simultaneously. Superior vena cava obstruction (SVCO) is a medical emergency and most often manifests in patients with a malignant disease process requiring immediate diagnostic evaluation and therapy due to its' life threatening presentation. The management of the SVCO is usually with chemotherapy, radiotherapy or intervention. In cases of large tumor burden, management of SVCO can trigger other complications. Tumor lysis syndrome is an oncologic emergency, which is characterized by a massive release of intracellular potassium, phosphate, and nucleic acid metabolites into the systemic circulation, which can be life-threatening. We present the case of a T-cell acute lymphoblastic leukemia with superior vena cava syndrome, developing tumor lysis syndrome on instituting definitive chemotherapy in a young patient.

Keywords: Superior Vena Cava Syndrome; Tumor Lysis Syndrome; T-Lymphoblastic Lymphoma; Oncological Emergencies.
\end{abstract}

\section{Introduction}

Superior vena cava obstruction (SVCO) and tumor lysis syndrome (TLS) are one of the most life-threatening oncologic emergencies. Lung cancer is the most common malignant cause of SVCO, while lymphoma, metastatic mediastinal tumours or lymph nodes, and indwelling catheters are often the other aetiologies [1].

Malignant SVCO can be caused by direct invasion of tumor into the SVC, or by external compression of the SVC by an adjacent pathologic process involving the right lung, lymph nodes, and other mediastinal structures, leading to stagnation of flow and thrombosis. Signs and symptoms resulting from obstruction of the superior vena cava include swelling of the upper body of the head, neck, arms, and/or breast and may also be associated with cyanosis, plethora, and distended subcutaneous vessels [2].

Tumor Lysis Syndrome is characterized by occurrence of hyperuricemia, hyperkalemia, hyperphosphatemia and hypocalcemia as a result of the destruction of a large number of rapidly proliferating neoplastic cells. It is usually associated with the initiation of chemotherapy but may occur with radiotherapy, surgery, endocrine therapy,

*Corresponding author: drtilaktvs@gmail.com

do) http://dx.doi.org/10.28991/SciMedJ-2021-0301-6

$>$ This is an open access article under the CC-BY license (https://creativecommons.org/licenses/by/4.0/).

(C) Authors retain all copyrights. 
glucocorticoids, interferon, hyperthermia or even spontaneously. There is potentially increased risk of the development of tumor lysis syndrome because of the rapid cell breakdown with the introduction of increasingly effective drugs and high dose chemotherapy regimens $[3,4]$.

Lymphoblastic lymphoma is a distinct entity and constitutes about 2-4\% of adult non-Hodgkin's lymphomas (NHLs) [5]. T- Lymphoblastic lymphoma (T-LBL) accounts for most cases of childhood NHL of T cell lineage [6]. They often present with bulky mediastinal masses, with associated pressure symptoms resulting in SVCO [7]. The bulky masses and bone marrow involvement are risk factors for tumor lysis syndrome [8].

In this Case-report, we present the occurrence of both the emergencies in a patient of T-lymphoblastic lymphoma/leukemia, which was successfully managed with anticipation and institution of appropriate therapy.

\section{Case Report}

A 20 year-old male with no known co-morbidities presented to the hospital with complaints of sub-acute onset, painless, progressive, bilateral diffuse swelling of the neck which was associated with hoarseness of voice, dyspnea on exertion, generalised weakness and significant weight loss 10-12 kgs of 03 weeks duration. The symptoms were not associated with chest pain, palpitation, paroxysmal nocturnal dyspnea [PND], orthopnoea, pre-syncope, syncope, any diurnal variation or cough, expectoration, fever, drenching night sweats, pruritus.

Examination revealed a young male with Eastern Cooperative Oncology Group [ECOG]-Performance Status [PS] of 3, temperature of $980 \mathrm{~F}$ and pulse of 132 / min- regular. The blood pressure was $142 / 90 \mathrm{~mm}$ of $\mathrm{Hg}$ with no postural fall and oxygen saturation of $86 \%$ at room air (98\% with Oxygen @ 6 ltrs/min) with respiratory rate of 26/ min. He had pallor but no icterus, cyanosis or pedal edema. He had bilateral, non-tender, mobile axillary lymph nodes $(1 \times 1 \mathrm{~cm}$; 1-2 in number). Fullness of neck was noted, which was diffuse, soft and non-pulsatile along with engorged tortuous veins on upper anterior chest wall (Figure 1).

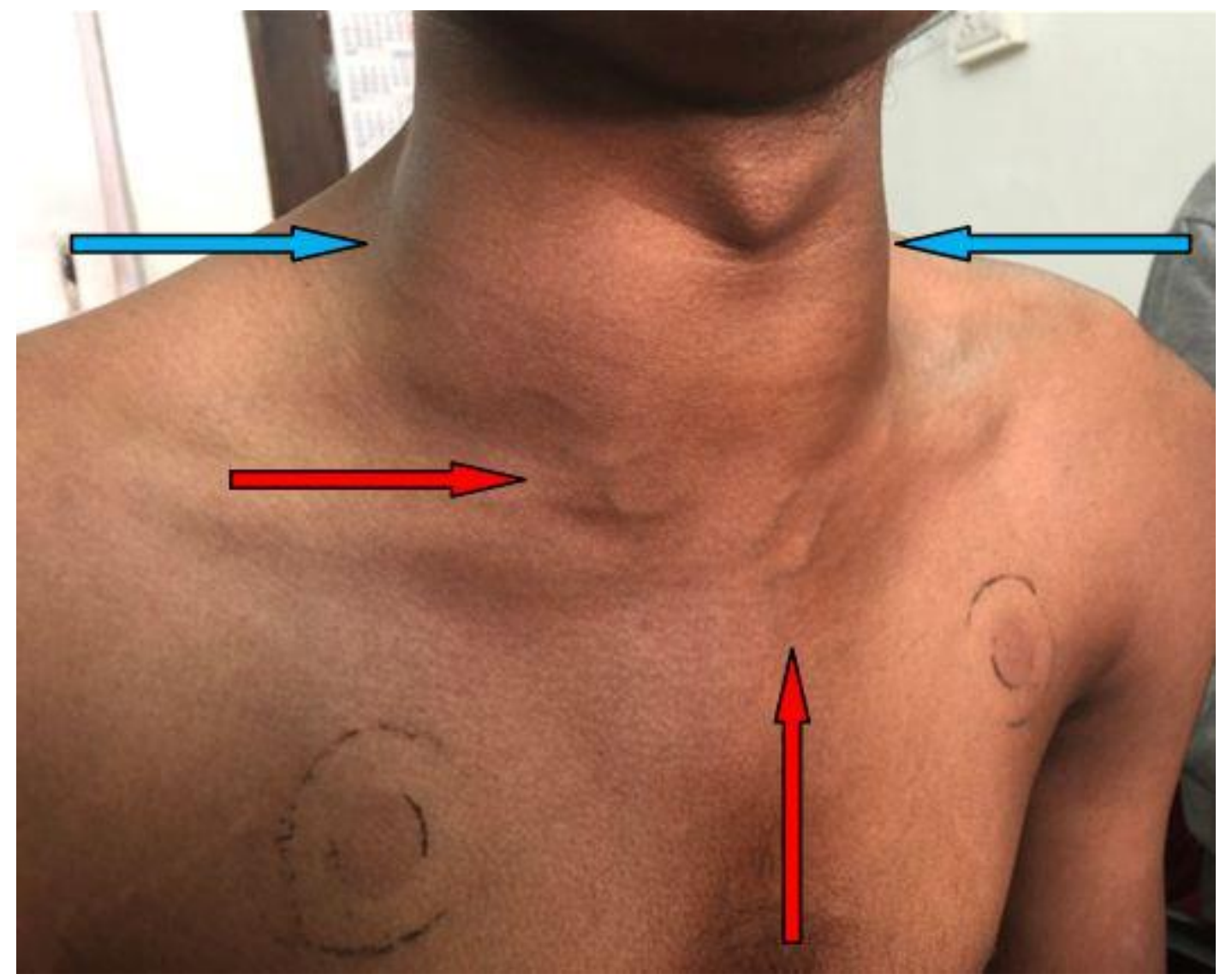

Figure 1. Neck swelling depicted by blue arrows; Prominent engorged veins depicted by red arrows, a presenting symptom of superior vena cava syndrome

The jugular venous pulsation (JVP) was not appreciable. Examination of abdomen revealed hepatomegaly of $3 \mathrm{~cm}$ and splenomegaly of $2 \mathrm{~cm}$. Respiratory system examination revealed reduced breath sound in right infrascapular region. Cardiovascular and neurological examination were unremarkable. The laboratory parameters at admission and during the course of admission are summarized in Table 1. 
Table 1. Laboratory evaluation at admission, at 48 hours and at 96 hours

\begin{tabular}{lcc}
\hline \multicolumn{1}{c}{ Investigations } & At Admission & $\mathbf{4 8 ~ h r s ~}$ \\
\hline Hemoglobin (g/dL) & 13.8 & 10.7 \\
TLC (per mm ${ }^{3}$ ) & 53,100 & 3,500 \\
DLC (Neutrophils \% / Lymphocyte \%) & $25 / 72$ & $30 / 64$ \\
Platelets / (per mm ${ }^{3}$ ) & 46,000 & 45,000 \\
Sodium / Potassium (mEq/L) & $135 / 4.1$ & $126 / 6.7$ \\
Calcium / Phosphate (mg/dL) & $7.9 / 2.8$ & $6.9 / 12.1$ \\
Urea (mg/dL) & 24 & 67 \\
Creatinine (mg/dL) & 1.2 & 1.2 \\
LDH (U/L) & 6453 & 8230 \\
Uric Acid (mg/dL) & 4.8 & 9.9 \\
Total Protein (gm/dL) & 7.5 & 7.1 \\
Albumin (gm/dL) & 4.1 & 3.6 \\
Bilirubin(Total/Direct) (mg/dL) & $0.3 / 0.1$ & $0.6 / 0.1$ \\
AST/ALT (IU/L) & $89 / 32$ & $293 / 36$ \\
ALP (IU/L) & 108 & 81 \\
\hline
\end{tabular}

TLC- Total Leucocyte Count; DLC- Differential Leucocyte Count; LDH- Lactate Dehydrogenase

AST- Aspartate Aminotransferase; ALT- Alanine Aminotransferase

The chest X-ray at admission revealed superior mediastinal widening with Right hilar prominence. A CECT scan of the neck and chest revealed a $14 \times 12 \mathrm{~cm}$ soft tissue mass in antero-superior mediastinum encasing the major vessels with associated right sided pleural effusion. (Figures $2 \mathrm{a}$ and $2 \mathrm{~b}$ ).
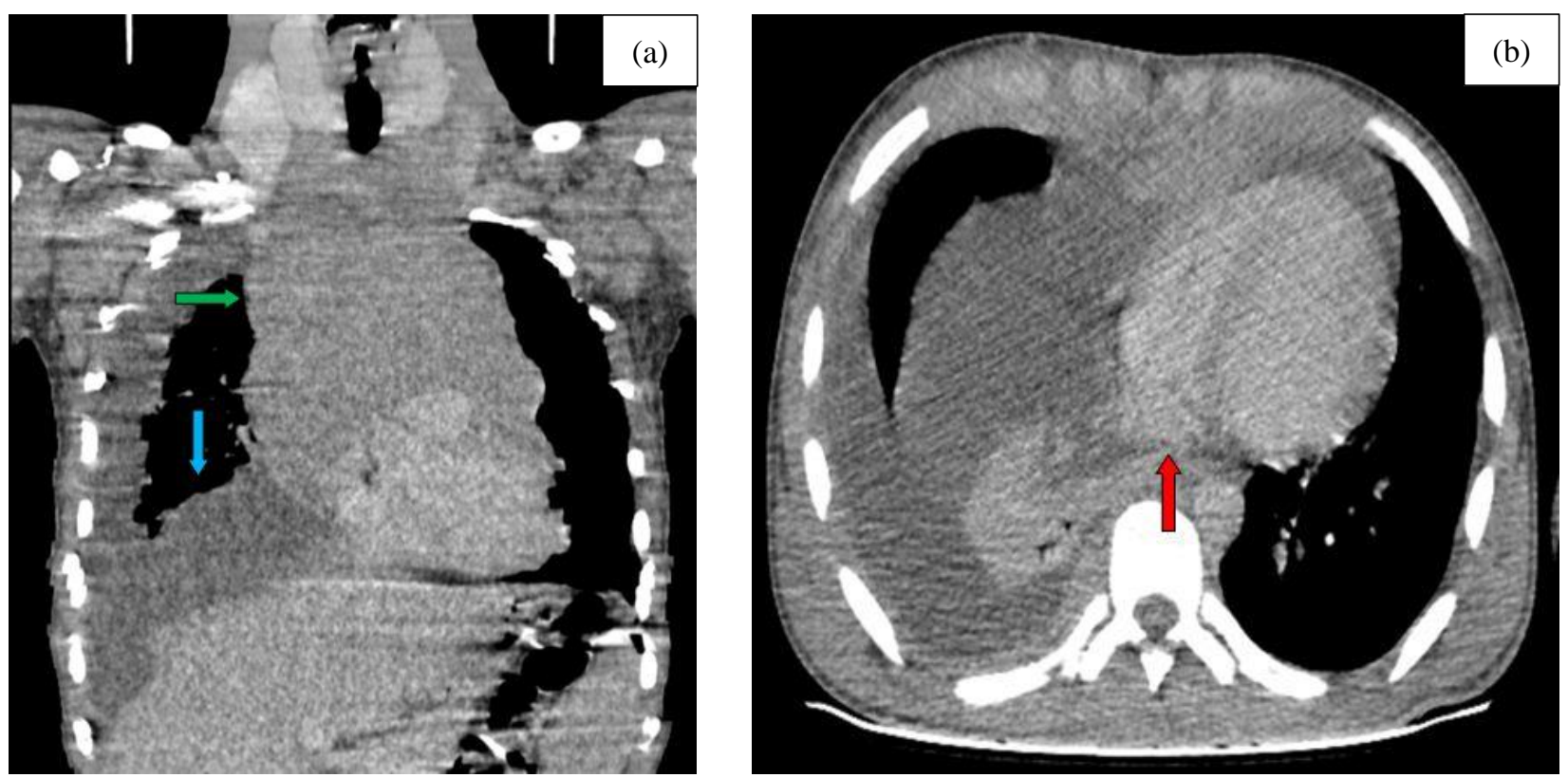

Figure 2a \& 2b. Chest CECT demosntrating a large tumour in the superior mediastinum (green arrow), with extensive lymphadenopathy and pleural effusion (blue arrow) almost completely compressing the superior vena cava (red arrow)

The bone marrow aspiration and biopsy revealed $90 \%$ blasts which were MPO Negative. Flow cytometry revealed features of Precursor T-Lymphoblastic Lymphoma [Bright Positive for CD3, CD7, CD4, CD8; Dim to Moderate positivity for CD1a, CD5, CD10, CD38 and Negative for CD19, CD20, CD13, CD33, CD117, MPO and HLA-DR] (Figure 3a, 3b, 3c, 3d, 3e, 3f). Cytogenetic analysis showed no analysable metaphases after 24hrs culture. Cerebrospinal fluid (CSF) was negative for blasts. 

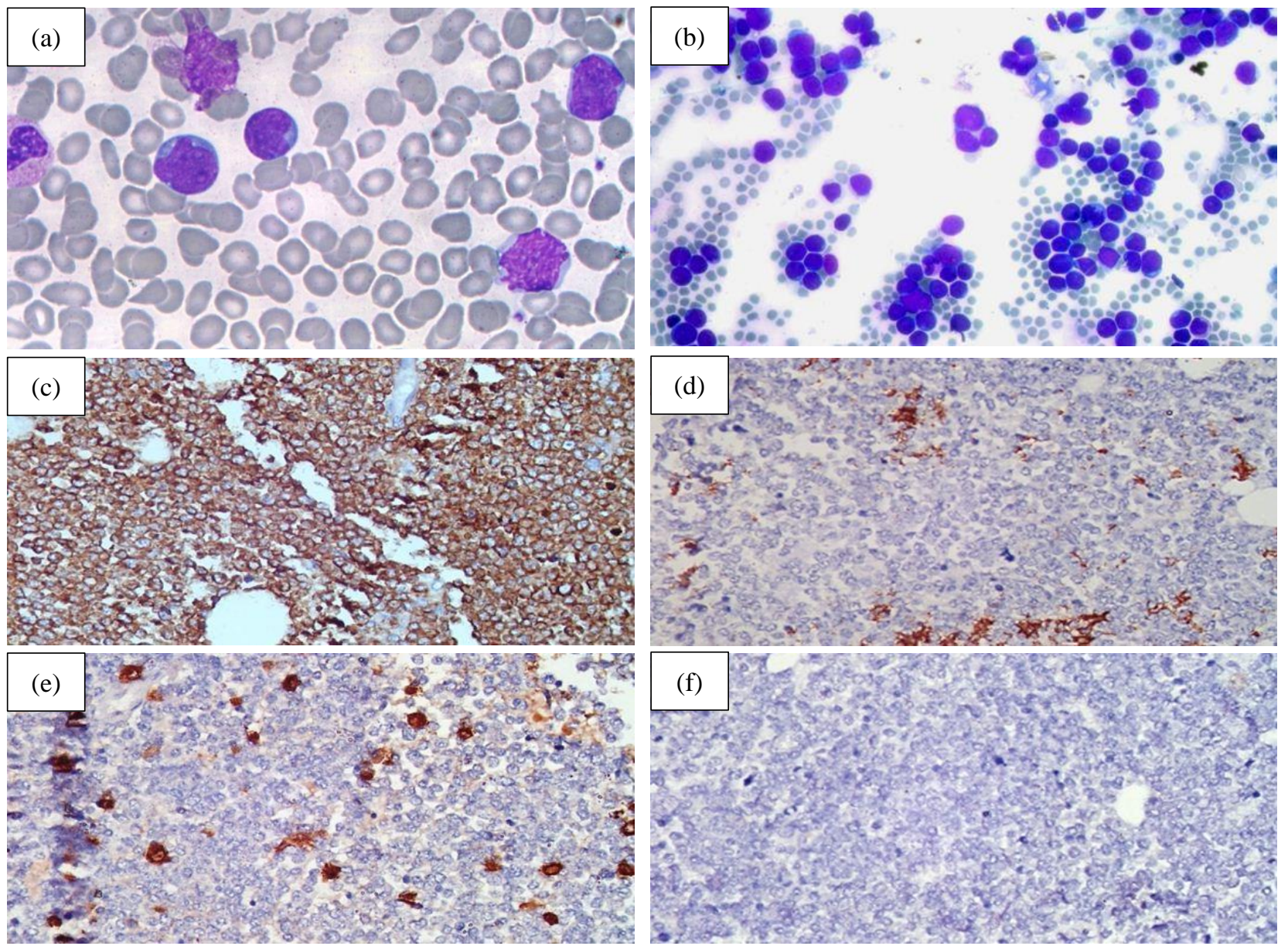

Figure 3. a) Peripheral blood smear with blasts having high N:C ratio and scant blue cytoplasm, as depicted by red arrows (Leishman Giemsa, 400x); 3b) Bone marrow aspirate showing aggregates of lymphoid blasts (Leishman Giemsa, 100x); 3c) IHC on BM biopsy section showing CD3 staining sheets of T lymphoid blasts(100x); 3d) Negative CD19 IHC on blasts(100x); 3e) Negative MPO staining (100x); 3f - Negative immunostaining for CD 117 (100x).

The diagnosis of Precursor T-cell Lymphoblastic Lymphoma/Leukemia was confirmed and patient was commenced on intravenous fluids and Tab Allopurinol $100 \mathrm{mg} 8$ hourly orally along with supportive care. He was started on Induction chemotherapy [Modified BFM-90 Lymphoblastic Lymphoma regime].

Evaluation after $24 \mathrm{hrs}$ of starting therapy revealed features of Laboratory TLS. Patient was managed with IV hydration, Inj Furosemide, Insulin-Glucose infusion, Salbutamol nebulization and Inj Rasburicase. Patient required blood and platelet support in view of anemia and thrombocytopenia. He showed clinical response over 48 hours and repeat laboratory evaluation confirmed normalization of TLS parameters. He was continued on induction as per protocol. Over the next two weeks, the features of SVC syndrome resolved with absence of neck swelling, improvement in voice quality and dyspnea. The course in the hospital was complicated by development of febrile neutropenia (culture negative), which responded to first-line intravenous antibiotics. Bone marrow evaluation postinduction revealed Minimal Residual Disease (MRD) of $<0.001 \%$. Presently he is asymptomatic and continuing the protocol therapy as planned and on regular follow up (Figure 4). 
Day 0

Initial presentation with Superior Vena Cava Obstruction

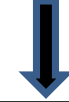

Day 1

Diagnosis of T-Lymphoblastic Lymphoma/ Leukemia confirmed and therapy instituted

Day 2

Tumor lysis syndrome detected and Rasburicase administered along with supportive therapy

Day 12

Superior vena cava obstruction resolved

Tumor lysis syndrome resolved

Chemotherapy and supportive care continued
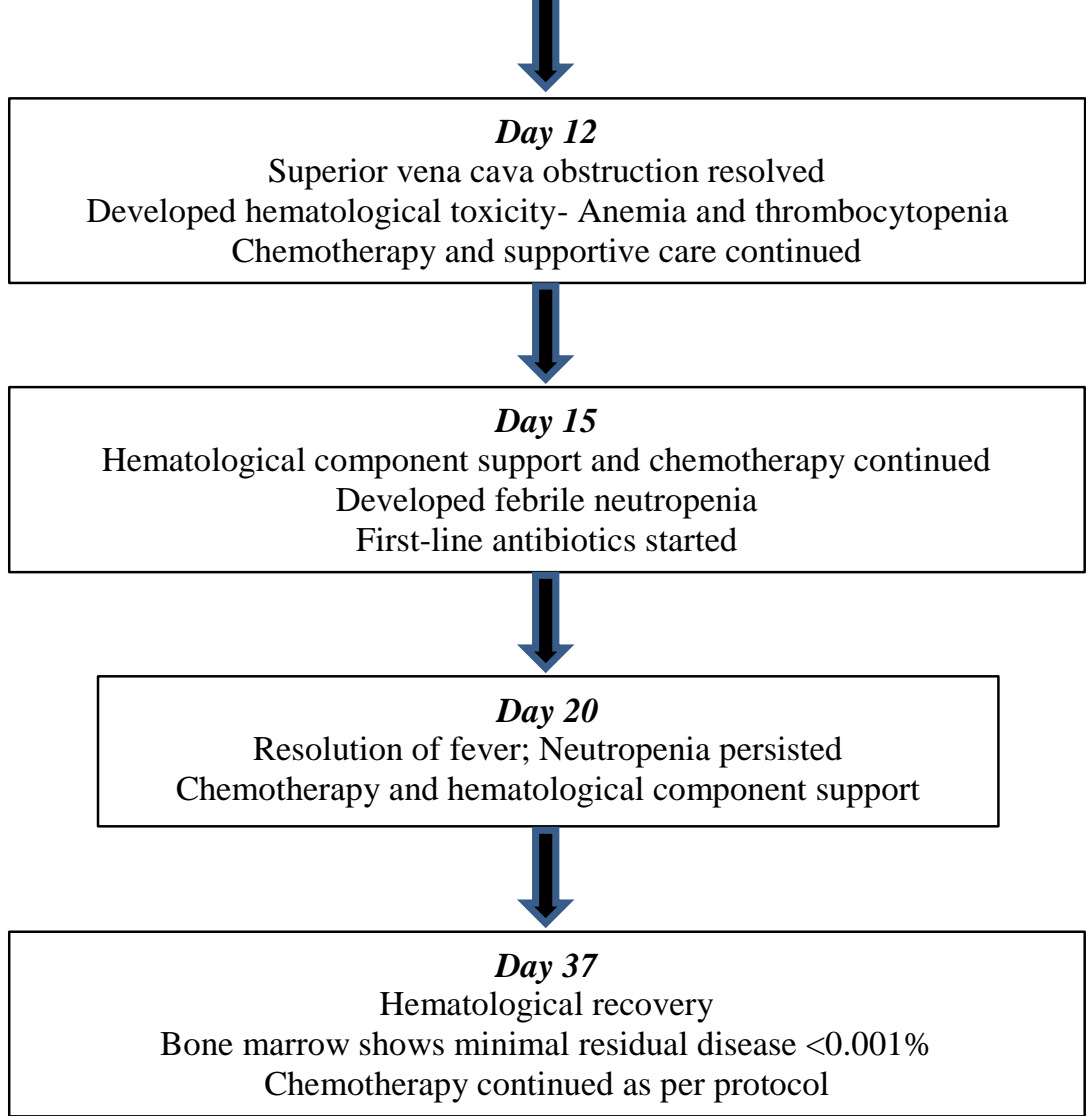

Figure 4. Flowchart depicting the course of events of the patient from the time of presentation till discharge

\section{Discussions}

T-LBL and T cell acute lymphoblastic leukemia (T-ALL) differ by the extent of bone marrow involvement, which is less than $20 \%$ in T- LBL [5]. T-LBL/T-ALL often presents with mediastinal mass resulting in shortness of breath, cough, and/or features of SVCO.

Superior vena cava syndrome requires high index of suspicion for early diagnosis and prompt therapy. The common symptoms include dyspnea, cough, cyanosis, facial plethora, distension of neck and chest wall veins and occasionally arm swelling [9]. These symptoms were seen in our case, as well. Our patient had clinical and radiological evidence of pleural effusion. This has been reported in literature with occurrence of SVCO [2]. Confirmatory diagnostic procedures including imaging modalities by CT and tissue histology are essential prior to 
starting definitive therapy. However, supportive therapy with positioning, oxygen, diuretics and steroids if indicated, is initiated at initial evaluation. Chemotherapy-sensitive malignancy respond rapidly (within weeks) to chemotherapy. However, for more chemo-resistant tumors, a combination of chemotherapy, radiotherapy and/or endovascular stenting may be considered [9]. In our case, the patient had a chemo-sensitive disease and responded well to chemotherapy and steroids and did not require any endovascular intervention. In an earlier study by the authors, a predictive model for event-free survival was derived using the clinical data of 55 patients with T-lymphoblastic lymphoma. The presence of poor performance status and SVCO at presentation had a hazard ratio of 6.2 towards poor event-free survival as compared either one of the features [10]. Our patient had both the adverse features at presentation. However, acute events in form of refractory disease or induction mortality did not occur. He had complete remission of the SVCO and achieved MRD at induction.

Tumor Lysis Syndrome, resulting from the rapid cell breakdown leading to the release of large quantities of potassium from the cytoplasm, urate from purine degradation and phosphate from nucleoproteins into the systemic circulation often occurs after initiation of chemotherapy in a patient with extensive tumor burden $[11,12]$. Our patient had significant risk of TLS in view of involvement of bone marrow and evidence of bulky disease, both established factors in literature [7]. The risk of TLS in this condition is the diagnostic criteria for TLS were laid down by Cairo and Bishop in 2004 [13]. Prophylactic IV fluid hydration and administration of Allopurinol or Rasburicase, initiated prior to chemotherapy is life-saving. Adequate hydration, use of allopurinol and strict monitoring of laboratory parameters enabled us to detect the development of TLS within 12 hours of initiating chemotherapy. The management of established TLS with Rasburicase and supportive measures enabled the patient to have a successful outcome. The dose of Rasburicase used in our case was a single dose of $3 \mathrm{mg}$, which is as effective as weight-based dosing, as described in various studies [14-16].

\section{Conclusion}

Oncological emergencies have myriad clinical presentations. They often co-exist in the same patient, making the management of the patient challenging. The possible emergencies in a patient can, at times, be anticipated in some malignancies with their clinical presentation and the course during therapy. We report a case of T-cell Acute Lymphoblastic lymphoma/leukemia presenting with symptomatic superior vena cava syndrome. The diagnosis of this condition is usually clinical, as was in our case. The establishment of diagnosis is often a challenge in solid tumors, obtaining the tissue as well as confirmation by histopathology. It is more facile with hematological malignancies. The institution of definitive therapy relieved the SVCO, which is often the case in malignant SVCO. The modality of therapy may vary from chemotherapy in hematological cancers to radiotherapy in solid tumors. Interventions like stenting may be necessary in some cases, while surgery is rarely resorted to in the current era.

The satisfactory outcome of initial therapy in our case, however, led to tumor lysis syndrome despite being on prophylactic uricosuric agent. The TLS was detected timely and managed with hydration, diuretics and rasburicase. There was resolution of the hyperuricemic state without any systemic complications. He also developed febrile neutropenia and hematological toxicity during the course of the illness. These were anticipated, detected timely and managed successfully, resulting in an overall favorable outcome.

\section{Declarations}

\subsection{Author Contributions}

Conceptualization, S.G. and TVSVGK.T.; methodology, S.G.; validation, D.M. and V.S.; resources, V.S.; writing — original draft preparation, S.G.; writing — review and editing, TVSVGK.T.; visualization, D.M.; supervision, TVSVGK.T. All authors have read and agreed to the published version of the manuscript.

\subsection{Funding}

The author(s) received no financial support for the research, authorship, and/or publication of this article.

\subsection{Acknowledgements}

The authors acknowledge the support of Department of Radiodiagnosis \& Interventional Radiology, AFMC, Department of Immuno-Hematology and Blood Transfusion, AFMC and the nursing staff for management of this case.

\subsection{Ethical Approval}

All subjects gave their informed consent for inclusion before they participated in the study. The study was conducted in accordance with the Declaration of Helsinki, and the protocol was approved by the Institutional Ethics Committee of Armed Forces Medical College, Pune, India (IEC/2020/84 dt 05 May 2020). 


\subsection{Data Availability Statement}

The data presented in this study are available on request from the corresponding author. The data are not publicly available due to [Data being maintained at Hospital of Armed Forces Medical Services of India].

\subsection{Conflict of Interest}

The authors declare that they have no known competing financial interests or personal relationships that could have appeared to influence the work reported in this paper.

\section{References}

[1] Friedman, T., Quencer, K., Kishore, S., Winokur, R., \& Madoff, D. (2017). Malignant Venous Obstruction: Superior Vena Cava Syndrome and Beyond. Seminars in Interventional Radiology, 34(04), 398-408. doi:10.1055/s-0037-1608863.

[2] Tamir, S., Friehmann, T., Ratzon, R., Livneh, N., Spectre, G., Atar, E., ... Leader, A. (2019). Computed Tomography Findings Associated With 30-Day Mortality in Patients With Malignant Superior Vena Cava Syndrome. Journal of Computer Assisted Tomography, 43(6), 912-918. doi:10.1097/rct.0000000000000934.

[3] Lorigan, P. C., Woodings, P. L., Morgenstern, G. R., \& Scarffe, J. H. (1996). Tumour lysis syndrome, case report and review of the literature. Annals of Oncology, 7(6), 631-636. doi:10.1093/oxfordjournals.annonc.a010682.

[4] Mirrakhimov, A. E., Voore P, Khan M., and Ali A. M. (2015). Tumor lysis syndrome: A clinical review. World Journal of Critical Care Medicine, 4(2), 130-138. doi:10.5492/wjccm.v4.i2.130.

[5] Kantarjian, H. M., Hoelzer, D., \& Larson, R. A. (2001). Advances in the treatment of adult acute lymphocytic leukemia, Part II. Hematology/Oncology Clinics of North America, 15(1).

[6] Reiter, A., Schrappe, M., Parwaresch, R., Henze, G., Müller-Weihrich, S., Sauter, S., ... \& Riehm, H. (1995). Non-Hodgkin's lymphomas of childhood and adolescence: results of a treatment stratified for biologic subtypes and stage--a report of the Berlin-Frankfurt-Münster Group. Journal of Clinical Oncology, 13(2), 359-372. doi:10.1200/JCO.1995.13.2.359.

[7] Gao, X. Z., Wei, J. G., Li, S. L., Han, J., Wang, G. N., \& Li, W. C. (2020). Mediastinal T lymphoblastic lymphoma/leukemia: clinicopathological and prognostic analyses of 61 cases. Zhonghua Bing Li Xue Za Zhi = Chinese Journal of Pathology, 49(6), 601-606. doi:10.3760/cma.j.cn112151-20190929-00538.

[8] Howard, S. C., Jones, D. P., \& Pui, C.-H. (2011). The Tumor Lysis Syndrome. New England Journal of Medicine, 364(19), 1844-1854. doi:10.1056/nejmra0904569.

[9] Hinton, J., Cerra-Franco, A., Shiue, K., Shea, L., Aaron, V., Billows, G., ... Lautenschlaeger, T. (2018). Superior vena cava syndrome in a patient with locally advanced lung cancer with good response to definitive chemoradiation: a case report. Journal of Medical Case Reports, 12(1): 301. doi:10.1186/s13256-018-1843-4

[10] Tilak, T. V. S. V. G. K., Raina, V., Kumar, L., Sharma, A., Sharma, M. C., Vishnubhatla, S., \& Bakhshi, S. (2013). Superior vena cava syndrome and poor performance status at presentation affect survival in mediastinal T-lymphoblastic lymphoma-a single institute experience from India. Annals of Hematology, 92(7), 917-923. doi:10.1007/s00277-013-1727-z.

[11] Cairo, M. S., Coiffier, B., Reiter, A., \& Younes, A. (2010). Recommendations for the evaluation of risk and prophylaxis of tumour lysis syndrome (TLS) in adults and children with malignant diseases: an expert TLS panel consensus. British Journal of Haematology, 149(4), 578-586. doi:10.1111/j.1365-2141.2010.08143.x.

[12] Wilson, F. P., \& Berns, J. S. (2014). Tumor Lysis Syndrome: New Challenges and Recent Advances. Advances in Chronic Kidney Disease, 21(1), 18-26. doi:10.1053/j.ackd.2013.07.001.

[13] Cairo, M. S., \& Bishop, M. (2004). Tumour lysis syndrome: new therapeutic strategies and classification. British Journal of Haematology, 127(1), 3-11. doi:10.1111/j.1365-2141.2004.05094.x.

[14] Jeon, Y.-W., Kwak, D.-H., Park, S.-S., Yoon, J.-H., Lee, S.-E., Eom, K.-S., .. Cho, S.-G. (2017). Effectiveness of Single-dose Rasburicase in Patients with Lymphoid Malignancies at a High Risk for Tumor Lysis Syndrome. Clinical Lymphoma Myeloma and Leukemia, 17(9), 595-603. doi:10.1016/j.clml.2017.06.027.

[15] Abousaud, M. I., Rush, M. C., \& Rockey, M. (2020). Assessment of rasburicase utilization for tumor lysis syndrome management in pediatric and adult patients in the inpatient and outpatient settings. Journal of Oncology Pharmacy Practice, 107815522094536. doi:10.1177/1078155220945368

[16] Alakel, N., Middeke, J. M., Schetelig, J., \& Bornhäuser, M. (2017). Prevention and treatment of tumor lysis syndrome, and the efficacy and role of rasburicase. OncoTargets and Therapy, Volume 10, 597-605. doi:10.2147/ott.s103864. 